\title{
Effect of Relaxin in Two Models of Renal Mass Reduction
}

\author{
Sandra L. Garbera,b Yelena Mirochnik ${ }^{d}$ Carolyn Brecklin ${ }^{b}$ \\ Leonid Slobodskoy d J ose A.L. Arruda ${ }^{a-c}$ George Dunea ${ }^{a, b, d}$
}

Divisions of Nephrology at a Cook County Hospital, bUniversity of Illinois College of Medicine,

'Chicago VA Health Care System and dHektoen Institute for Medical Research, Chicago, III., USA

\section{Key Words}

Growth factors · Relaxin - Renal mass reduction . Glomerulosclerosis

\begin{abstract}
Background: Relaxin (RIx), a 6-kD protein hormone, belongs to the insulin growth factor family. We have previously shown that RIx reduces interstitial fibrosis in a model of chronic papillary necrosis. Hypothesis: The purpose of this study was to extend these observations to a model of renal injury induced by mass reduction. Material and Methods: Renal mass was reduced by either infarction or surgical excision of both poles, with removal of the contralateral kidney. Two weeks later, creatinine clearance was done and animals from both groups implanted with osmotic pumps delivering either Rlx $(2 \mu \mathrm{g} / \mathrm{h})$ or vehicle (Veh). Treatment was continued for 4 weeks. The severity of the glomerular injury was quantified by planimetric measurements. Renal function was assessed by creatinine clearance and plasma creatinine. Results: RIx significantly decreased systolic blood pressure in animals with infarction. This was accompanied by a decrease in serum creatinine and a slight improvement in creatinine clearance. The severity of the glomerular lesion was reduced by $\mathrm{Rlx}$ (sclerosis index, Veh $1.16 \pm 0.13$ vs. RIx $0.74 \pm 0.16, p=0.037)$. In the excision group the animals
\end{abstract}

\section{KARGER}

Fax +4161306 1234 E-Mail karger@karger.ch www.karger.com

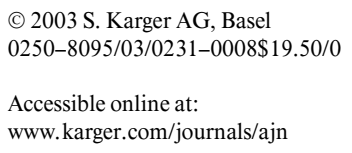

were normotensive. In this group, RIx treatment was accompanied by a decrease in serum creatinine (Veh 1.01 \pm 0.03 vs. $R I x 0.81 \pm 0.05 \mathrm{mg} / \mathrm{dl}, \mathrm{p}=0.02$ ) and an increase in GFR (Veh $0.90 \pm 0.14$ vs. RIx $1.33 \pm 0.11 \mathrm{ml} / \mathrm{min}, \mathrm{p}=$ 0.03). The sclerosis index was also reduced. Conclusion: RIx decreases renal injury by at least two mechanisms, one by lowering blood pressure as seen in the infarction model, the other independent of blood pressure as seen in the normotensive excision model where there was also a significant functional improvement.

Copyright $\odot 2003$ S. Karger AG, Basel

\section{Introduction}

Relaxin (Rlx), a member of the insulin-like growth factor family, has numerous biological effects [for a review, see Bani 1]. It has been shown to be a potent vasodilator in the kidney [2, 3] as well as in the coronary circulation [4]. In an earlier study we found that Rlx decreased renal interstitial fibrosis in a model of chemically induced papillary necrosis, apparently independent of systemic hemodynamic changes [5].

Renal mass reduction may be achieved either by excision (Exc) or infarction (Inf) of the kidney. In the Inf model the animals develop a striking hypertension and a severe glomerular lesion. In the Exc model, hypertension 
Table 1. Effect of Rlx on systolic blood pressure and urinary volume

\begin{tabular}{lrlll}
\hline & $\mathrm{n}$ & $\begin{array}{l}\text { Systolic blood } \\
\text { pressure at 2 weeks }\end{array}$ & $\begin{array}{l}\text { Systolic blood } \\
\text { pressure at 6 weeks }\end{array}$ & $\begin{array}{l}\text { Urinary volume } \\
\text { ml/h at 6 weeks }\end{array}$ \\
\hline Inf no Rlx & 14 & $169 \pm 8$ & $169 \pm 7$ & $1.46 \pm 0.10^{* *}$ \\
Inf + Rlx & 10 & $175 \pm 6$ & $146 \pm 7^{*}$ & $1.85 \pm 0.19^{* *}$ \\
Exc no Rlx & 4 & $123 \pm 6$ & $129 \pm 9$ & $0.74 \pm 0.12$ \\
Exc + Rlx & 7 & $129 \pm 5$ & $128 \pm 7$ & $0.87 \pm 0.09$ \\
Intact S-D rat & 15 & $127 \pm 9$ & $126 \pm 6$ & $0.61 \pm 0.10$ \\
\hline
\end{tabular}

$* \mathrm{p}<0.04$ compared to the untreated Inf group; $* * \mathrm{p}<0.01$ compared to intact rats and the Exc group. does not appear in the first month and the glomerular lesion has been reported to be less severe. The models are comparable in single nephron blood flow and glomerular filtration rate (GFR) in the remnant kidney at 1 month [6]. The purpose of this study was to evaluate the effect of Rlx on glomerular structure and function in the presence or absence of hypertension.

\section{Material and Methods}

\section{Experimental Design}

Male Sprague-Dawley rats (Harlan) weighing $350 \mathrm{~g}$ at the beginning of the study were housed individually on a standard 12:12 h light-dark cycle with free access to standard rat chow (PMI \#5012; Purina Mills Feeds, Inc., St. Louis, Mo., USA) and water. The animals were conditioned to handling and tail-cuff blood pressure measurement (IITC Life Science, Woodland Hills, Calif., USA) for 2 weeks before surgery. All animals had stable blood pressure at that time. The experiments were conducted in accord with the NIH guidelines for the care and use of laboratory animals and approved by the Institutional Review Board.

Renal Mass Reduction. The procedures used for renal mass reduction were essentially those of Griffin et al. [6]. In the Inf model the superior and inferior branches of the left renal artery were ligated and the right kidney was removed (Inf). In the second model (Exc) the superior and inferior poles of the left kidney were surgically removed followed by right nephrectomy. Two weeks later, animals were placed in metabolic cages and 24-hour urine specimens were collected for creatinine clearance. Following sample collection, osmotic mini-pumps (Alzet model 2ML4; Durect Corp., Cupertino, Calif., USA) filled with either Rlx (recombinant human relaxin, ConXn ${ }^{\mathrm{TM}}, 2 \mu \mathrm{g} / \mathrm{h}$; Connetics Corp., Palo Alto, Calif., USA) or vehicle (Veh) $(20 \mathrm{~m} M$ sodium acetate, $\mathrm{pH}$ 5.0) were implanted subcutaneously between the scapulae. Drug delivery continued for 4 weeks. Blood pressures were measured twice weekly during this period. At day 28, another 24-hour urine collection was obtained for creatinine clearance. The rats were anesthetized with sodium pentobarbital $(50 \mathrm{mg} / \mathrm{kg})$ and the kidney perfused retrogradely with cold saline followed by HistoChoice (Amresco Inc., Solon, Ohio, USA). Blocks of tissue distant from the lesion were processed for histology and immunohistochemistry.

Relaxin in Glomerular Sclerosis

\section{Biochemical Studies}

Urine and plasma creatinine were measured by the method of Heingård and Tiderström [7] using a kit obtained from Sigma Diagnostics (Sigma, St. Louis, Mo., USA). GFR was assessed by creatinine clearance in conscious animals, a method previously documented in our laboratory to be an accurate reflection of GFR [8]. Urine albumin was measured by Albumin Blue 580 using a rat albumin standard [9].

\section{Immunohistochemistry}

Collagen IV antibodies were purchased from Southern Biotechnology Associates Inc. (Birmingham. Ala., USA). Macrophages were identified with a mouse monoclonal antibody to ED-1 (Harlan Bioproducts, Indianapolis, Ind., USA). In all cases the secondary antibody was conjugated to alkaline phosphatase and visualized with Fast Red Naphthol (Sigma).

\section{Morphometric Analysis}

Glomerular lesions were evaluated on periodic acid-Schiff (PAS)stained digitized sections. Fifty glomeruli distant from the point of insult were photographed at $10 \times$ and digitized. The PAS-positive area was measured by means of Sigma Scan (SPSS, Chicago, Ill., USA) and expressed as a percent of the total area of that glomerulus. Measurements were then grouped using the sclerosis index of Cao et al. [10] as follows: 0 , normal; 1 , mesangial expansion and slight glomerular damage involving $<25 \%$ of the glomerulus; 2 , mild sclerosis $25-50 \% ; 3$, moderate $50-75 \%$, and 4 severe, $>75 \%$ involvement.

\section{Statistics}

Unless otherwise indicated, data are expressed as mean \pm SE. Statistical analyses (paired or unpaired t-tests) were performed where appropriate with Sigma Stat (SPSS).

\section{Results}

Renal Inf induced severe hypertension that was ameliorated by Rlx (table 1). Rlx had no effect on systemic blood pressure in animals whose renal mass was reduced by Exc. There was a significant increase in the 24-hour urine output in the Inf rats, which was further increased in the animals receiving Rlx. Rlx treatment had no effect on 


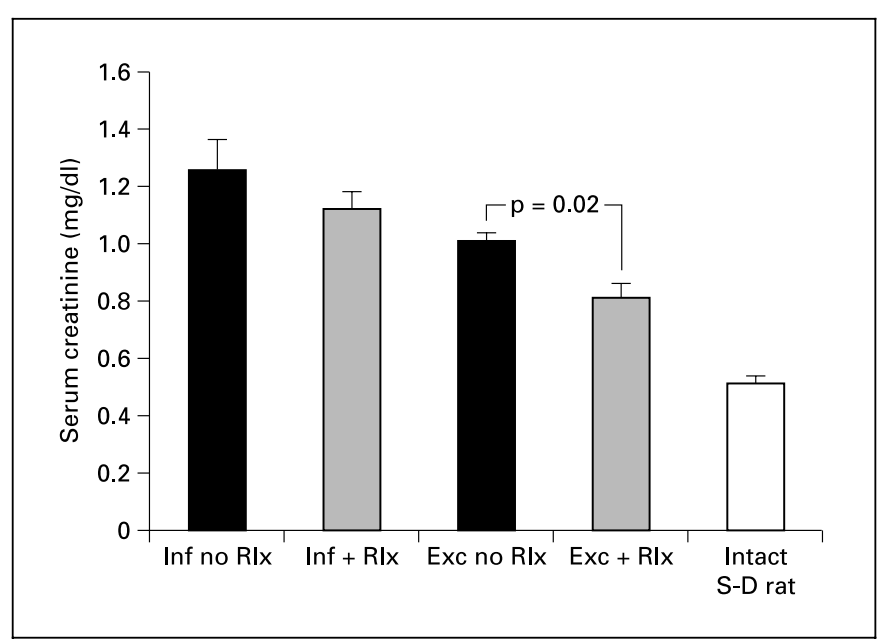

Fig. 1. Serum creatinine levels at 6 weeks after renal mass reduction and following 4 weeks of Rlx treatment. Intact rats are included for comparison purposes.

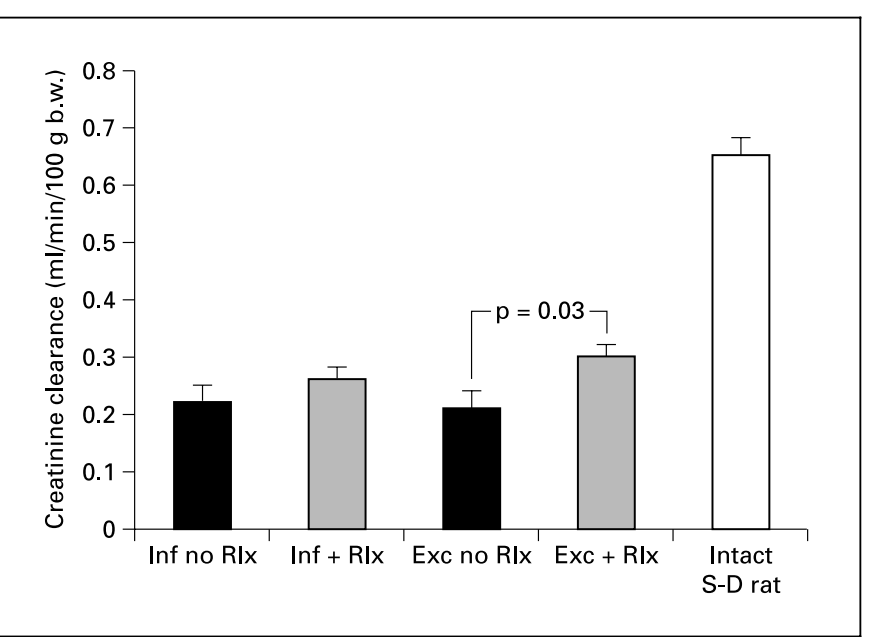

Fig. 2. Creatinine clearance levels at 6 weeks after renal mass reduction and following 4 weeks of Rlx treatment. Intact rats are included for comparison purposes.

urinary albumin excretion; however, there was a difference in albumin excretion in the two models (Inf $163 \pm$ 32 vs. Exc $10 \pm 1 \mathrm{mg} / 24 \mathrm{~h}$ ).

Two weeks after mass reduction, serum creatinine was elevated in both models in comparison to intact rats (Inf $1.10 \pm 0.04$,Exc $0.87 \pm 0.03$, intact $0.58 \pm 0.04 \mathrm{mg} / \mathrm{dl}$ ). Following 1 month of Rlx treatment, creatinine was significantly reduced only in the Exc group (fig. 1) but Rlx prevented the rise in serum creatinine in both models. These changes were reflected in the creatinine clearance

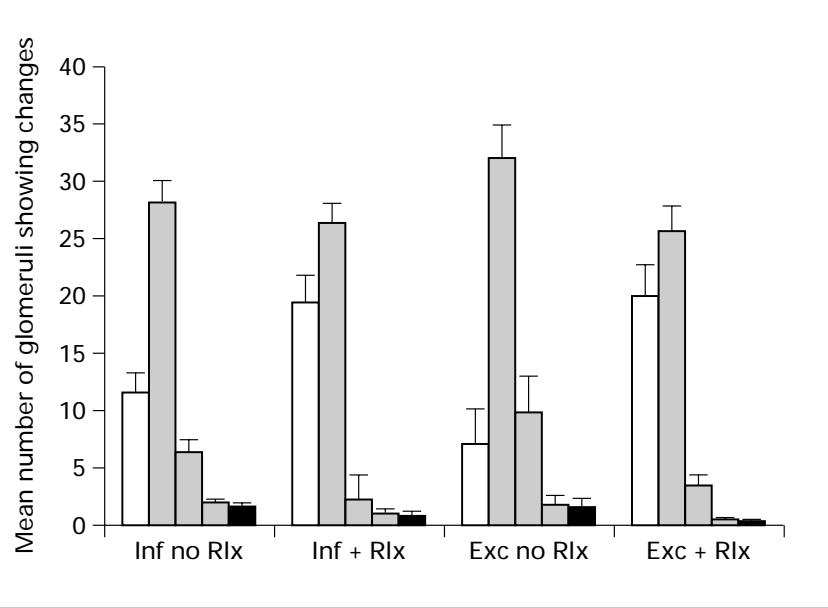

Fig. 3. Extent of glomerular lesions in Inf and Exc models 6 weeks after renal mass reduction. $\square=$ Normal; ; = slight; $\square=$ mild; $\mathbb{\square}$ = moderate; $\mathbf{\square}=$ severe.

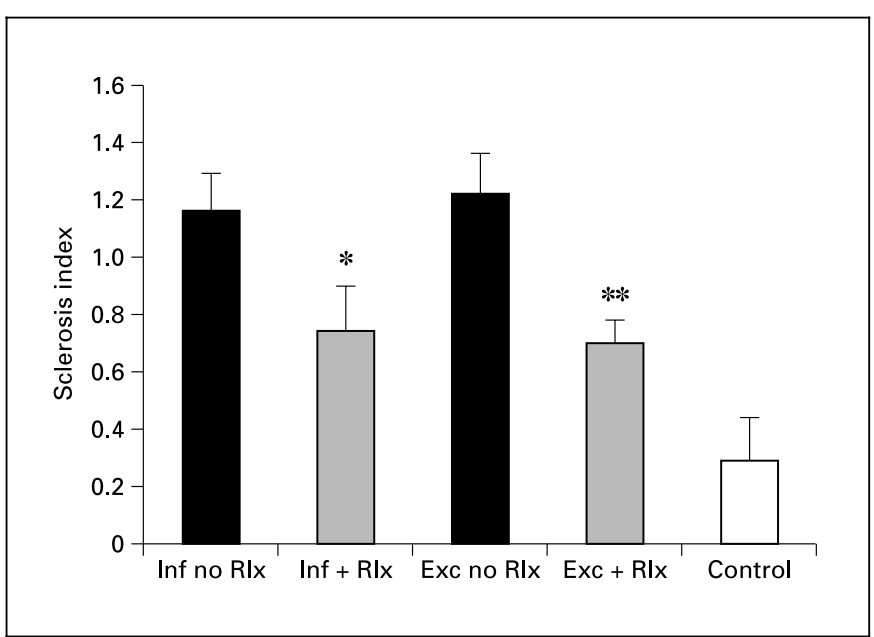

Fig. 4. Glomerulosclerosis index. ${ }^{*} \mathrm{p}<0.05$ and ${ }^{* *} \mathrm{p}<0.005$ vs. untreated animals.

where there was a significant improvement in the Exc group (fig. 2). In the Inf rats, Rlx prevented the decrease in creatinine clearance ( Inf pre $0.27 \pm 0.02$, Inf post 0.22 \pm 0.03 , Inf post + Rlx $0.26 \pm 0.02 \mathrm{ml} / \mathrm{min} / 100$ g b.w.).

The distribution of the glomerular lesions is depicted in figure 3. In the Inf model, Rlx caused a decrease in the number of glomeruli showing mild to severe sclerotic changes as well as an increase in the number of normal glomeruli. In the Exc model the lesion was slightly more severe as noted by the increased number of glomeruli with 
Fig. 5. Collagen IV staining: A normal rat, B Inf, no treatment, C Inf + Rlx, and D Exc + Rlx.

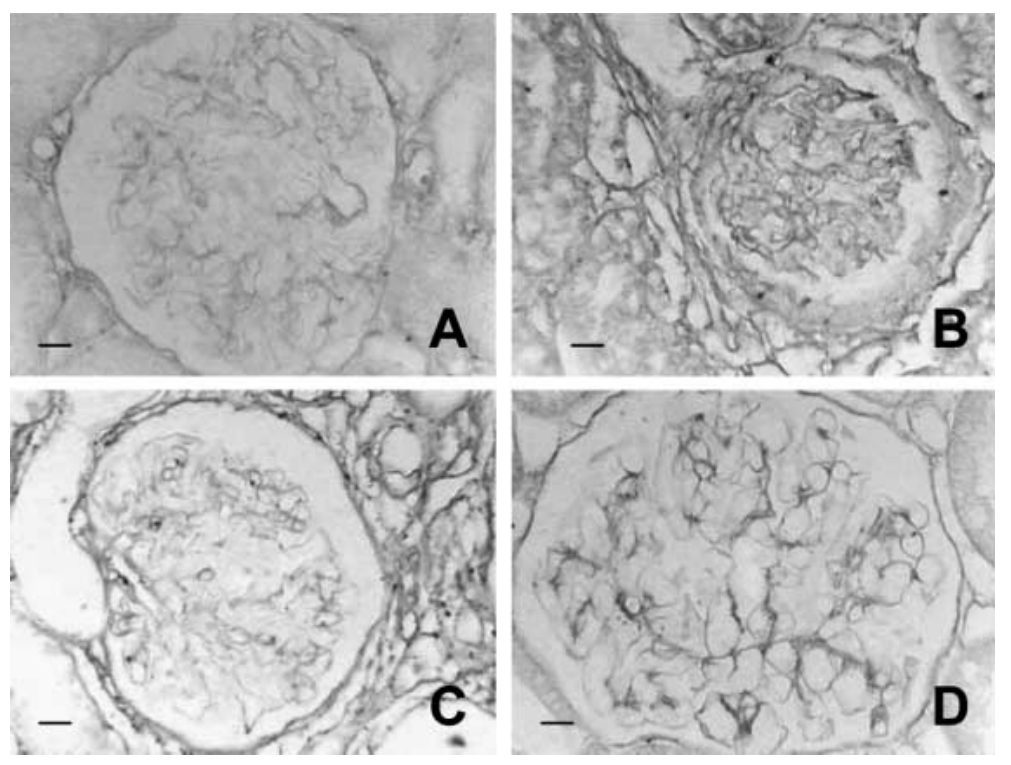

slight to mild lesions. Rlx caused a more dramatic shift in this lesion with $60 \%$ increase in the number of unaffected glomeruli and a $58 \%$ decrease in the number of glomeruli showing mild to severe changes. These changes are summarized and compared as the sclerosis index in figure 4.

Collagen IV as visualized by immunohistochemistry was markedly increased in both models. Rlx decreased the deposition of collagen IV within most glomeruli (fig. 5). With the exception of the increased density of collagen IV around the capillary loops in the Inf model, many of the glomeruli had a normal appearance. Interstitial collagen IV did not appear to be decreased in this model. In the Exc model, collagen IV was decreased in the glomeruli, accompanied by a decrease in interstitial collagen IV.

\section{Discussion}

Rlx and its relative, Rlx-like factor (RLF/INSL3), are structurally related to the insulin growth factor family. Its functions in pregnancy, including softening of the cervix at parturition, inhibition of uterine contractions, and growth of the mammary gland have been known for some time and are well documented [11]. In addition to its function in reproduction, Rlx is a paracrine hormone found in neovascularization during wound healing [12]. It increases coronary blood flow and counteracts the myocardial damage induced by ischemia-reperfusion in the isolated guinea pig heart $[4,13]$. These effects of $\mathrm{Rlx}$ are supported by studies showing Rlx binding to the rat atria, where the receptor density was actually higher than in the uterus [14].

Studies have also shown that Rlx has a potent effect on the vasculature of the liver [15] and lung [16]. In the kidney, Rlx increases renal blood flow without an effect on systemic blood pressure [2]. This effect of Rlx is probably mediated by endothelin and nitric oxide [3].

Rlx also functions as an antifibrotic agent. In human dermal fibroblasts, Rlx modulates collagen deposition by decreasing collagen synthesis and increasing its degradation [17]. In human lung fibroblasts, Rlx inhibited TGF$\beta$-induced procollagen and fibronectin overexpression and has been associated with increased collagenase activity [18]. Williams et al. [19] reported that Rlx reduced effective collagen deposition in vitro and decreased liver fibrosis in vivo in a carbon tetrachloride $\left(\mathrm{CCl}_{4}\right)$ model. The change in collagen deposition was accompanied by a decrease in the metalloproteinase inhibitors TIMP-1 and TIMP-2. Rlx also decreased the nephrotoxicity seen following cyclosporine administration to rats [20].

We have previously shown that Rlx treatment improves renal structure and function in a model of papillary necrosis associated with interstitial fibrosis [5]. In the current study, renal mass reduction by Exc was accompanied by an increase in the serum creatinine and a fall in GFR. Few glomeruli showed severe sclerosis but many had pre-sclerotic changes as evidenced by an increase in cellularity and expansion of the mesangial matrix. Immunohistochemical staining showed that most of this in- 
creased matrix was composed of collagen IV. The rats had a normal blood pressure and their urine flow rate was normal. In this model, administration of Rlx for 4 weeks resulted in a significant improvement in renal function and a decrease in the number of glomeruli showing presclerotic changes. There was also a decrease in the amount of collagen IV.

Renal mass reduction by Inf produced a similar lesion accompanied by a markedly increased blood pressure and urine flow rate. The effect of Rlx in this model was less straightforward. In keeping with Rlx's known vasodilatory effect, there was a significant decrease in blood pressure. The histological appearance of the kidney was improved, as shown both by the morphometric measurements and the decreased collagen staining. The changes in renal function, however, were minimal.

There are several possible explanations for a lack of a significant improvement in renal function in the Inf model. Serum creatinines done 2 weeks after renal mass reduction and prior to Rlx infusion showed that the Inf group had a more severe functional impairment (SCr $1.10 \pm$
0.04 vs. Exc $0.87 \pm 0.03 \mathrm{mg} / \mathrm{dl}, \mathrm{p}<0.001)$. It is possible that at this dosage Rlx may only be adequate to lower blood pressure and prevent a worsening of the renal function.

In conclusion, Rlx improved GFR in the Exc model. Rlx decreased the severity of the histological lesion in both groups, as evidenced by a decrease in collagen IV and by morphometric measurements. Rlx lowered blood pressure in the hypertensive animals but had no effect in the Exc group. Thus, Rlx exerts a protective effect not only in the renal papillary necrosis model but also in those models associated with total nephron mass reduction. Rlx protects renal integrity both functionally and histologically in these two models. These changes are accounted for only in part by blood pressure reduction.

\section{Acknowledgements}

The authors would like to thank Dr. Elaine Unemori and Connetics Corporation for their generous gift of recombinant human relaxin and Dr. Sethupathi for the surgical preparations.

\section{References}

1 Bani D: Relaxin: A pleiotropic hormone. Gen Pharmacol 1997;28:13-22.

2 Danielson LA, Sherwood OD, Conrad KP: Relaxin is a potent renal vasodilator in conscious rats. J Clin Invest 1999;103:525-533.

3 Danielson LA, Kercher LJ, Conrad KP: Impact of gender and endothelin on renal vasodilation and hyperfiltration induced by relaxin in conscious rats. Am J Physiol 2000;279:R1298R1304.

4 Bani Sacchi T, Bigazzi M, Bani D, Mannaioni PF, Masini E: Relaxin-induced increased coronary flow through stimulation of nitric oxide production. Br J Pharmacol 1995;116:1589_ 1594.

5 Garber SL, Mirochnik Y, Brecklin C, Unemori EN, Singh AK, Grove BH, Slobodskoy L, Arruda JAL, Dunea G: Relaxin decreases renal interstitial fibrosis and slows progression of renal disease. Kidney Int 2001;59:876-882.

6 Griffin KA, Picken MM, Churchill M, Churchill $\mathrm{P}$, Bidani AK: Functional and structural correlates of glomerulosclerosis after renal mass reduction in the rat. $\mathbf{J}$ Am Soc Nephrol 2000;11:497-506.

7 Heinegård D, Tiderström G: Determination of serum creatinine by a direct colorimetric method. Clin Chim Acta 1973;43:305-310.

8 Garber SL, Mirochnik Y, Desai SS, Arruda JAL, Dunea G: Angiotensin-converting enzyme inhibition reduces the effect of bromoethylamine-induced papillary necrosis and renal fibrosis. J Am Soc Nephrol 1998;9:10521059
9 Pegoraro AA, Peracha W, Hasnain M, Ranginwala N, Shaykh M, Singh AK, Arruda JAL, Dunea G: Evaluation of a new fluorescent dye method to measure urinary albumin in lieu of urinary total protein. Am J Kidney Dis 2000; 35:739-744.

10 Cao Z, Cooper ME, Wu LL, Cox AJ, JandeleitDahm K, Kelly DJ, Gilbert RE: Blockade of the renin-angiotensin and endothelin systems on progressive renal injury. Hypertension 2000;36:561-568.

11 Sherwood OD: Relaxin; in Knobil E, Neill J (eds): The Physiology of Reproduction, ed 2. New York, Raven Press, 1993, vol 1, pp 8611010.

12 Unemori EN, Lewis M, Constant J, Arnold G, Grove BH, Normand J, Deshpande U, Salles A, Pickford LB, Erikson ME, Hunt TK, Huang $\mathrm{X}$ : Relaxin induces vascular endothelial growth factor expression and angiogenesis selectively at wound sites. Wound Repair Regen 2000;8: 361-370.

13 Masini E, Bani D, Bello MG, Bigazzi M, Mannaioni PF, Bani Sacchi T: Relaxin counteracts myocardial damage induced by ischemia-reperfusion in isolated guinea pig hearts: Evidence for an involvement of nitric oxide. Endocrinology 1997;138:4713-4720.

14 Tan YY, Wade JD, Tregear GW, Summers RJ: Quantitative autoradiographic studies of relaxin binding in rat atria, uterus and cerebral cortex: Characterization and effects of oestrogen treatment. Br J Pharmacol 1999;127:91-98.
15 Bani D, Nistri S, Quattrone S, Bigazzi M, Bani Sacchi T: The vasorelaxant hormone relaxin induces changes in liver sinusoid microcirculation: A morphologic study in the rat. J Endocrinol 2001;171:541-549.

16 Dschietzig T, Richter C, Bartsch C, Bohme C, Heinze D, Ott F, Zartnack F, Baumann G, Stangl K: Flow-induced pressure differentially regulates endothelin-1, urotensin II, adrenomedullin and relaxin in pulmonary vascular endothelium. Biochem Biophys Res Commun 2001; 289, 245-251.

17 Unemori EN, Amento EP: Relaxin modulates synthesis and secretion of procollagenase and collagen in human dermal fibroblasts. J Biol Chem 1990;265:10681-10685.

18 Unemori EN, Pickford LB, Salles AL, et al: Relaxin induces an extracellular matrix-degrading phenotype in human lung fibroblasts in vitro and inhibits lung fibrosis in a murine model in vivo. J Clin Invest 1996;98:2739_ 2745.

19 Williams EJ, Benyon RC, Trim N, Hadwin R, Grove BH, Arthur MJ, Unemori EN, Iredale JP: Relaxin inhibits effective collagen deposition by cultured hepatic stellate cells and decreases rat lever fibrosis in vivo. Gut 2001;49: 577-583.

20 Huang X, Cheng Z, Sunga J, Unemori E, Zsebo $\mathrm{K}$ : Systemic administration of recombinant human relaxin ameliorates the acute cyclosporine nephrotoxicity in rats. J Heart Lung Transplant 2001;20:253. 